\title{
HOLOGRAPHY AND INFRARED CONFORMALITY IN TWO DIMENSIONS
}

\author{
Marcus Berg \\ Università di Roma "Tor Vergata" \\ via della Ricerca Scientifica, 1 \\ 00133 Rome, Italy \\ berg@roma2.infn.it
}

\begin{abstract}
This is a very brief review of some results from Refs. [2] and [3]. In holographic renormalization, we studied the RG flow of a $2 \mathrm{~d} N=(4,4)$ CFT perturbed by a relevant operator, flowing to a conformal fixed point in the IR. Here, the supergravity dual is displayed, and the computation of correlators is discussed. The sample stress-energy correlator given here provides an opportunity to explicitly compare Zamolodchikov's $C$ function to the proposal for a "holographic $C$-function".
\end{abstract}

First, I will recall how to compute correlators holographically, even in the presence of domain walls (for a review, see [1]).

As a simple analogy to keep in mind, consider a medieval castle where soldiers are practicing cannon-firing from atop the castle walls into the interior courtyard. Let us say that when the wall has height $h_{0}$, a horizontally fired cannon ball hits the ground precisely in the middle of the courtyard. A priori the height of the wall $h$ and the angle of firing $\theta$ would of course be independent boundary conditions, but by requiring that the cannon ball always hit the center of the courtyard, these two become related: $h=h_{0} / \cos ^{2} \theta-r \tan \theta$, for a circular wall of radius $r$, and neglecting air resistance. For an uneven castle wall, where the height of the wall varies as $h(x)$ with the distance $x$ around the circumference of the castle, the same relation relates $\theta(x)$ to $h(x)$. Going back to $\mathrm{AdS} / \mathrm{CFT}$, the requirement that a bulk field $\phi$ vanishes in the deep interior relates a radial derivative at the boundary $\phi_{(1)}(x)$ to the boundary value $\phi_{(0)}(x)$. This relation encodes the desired boundary correlator 
(up to contributions from local counterterms):

$$
\langle\mathcal{O}(x) \mathcal{O}(y)\rangle \sim \frac{\delta \phi_{(1)}(x)}{\delta \phi_{(0)}(y)}
$$

and in fact, in the approximation of linear fluctuations around the domain wall, we will have $\phi_{(1)}(x)=f(x) \phi_{(0)}(x)+\mathcal{O}\left(\phi_{(0)}^{2}\right)$, and the correlator (1.1) is simply the relation $f(x)$ between the radial derivative and the boundary field, enforced by the requirement that the field vanish at a point in the deep interior.

To finish up this introductory part, I should admit that apart from the more obvious deficiencies of the above mechanical analogy, it is important that in general (for non-marginal operators) the problem we are actually solving is a scaled Dirichlet problem, such that one has to factor out some radial dependence to obtain a finite boundary value.

To get to the main result presented in this contribution - a stressenergy tensor correlator - I need to briefly review the analytic domain wall solution of [2] which interpolates between two AdS vacua. It was constructed as a solution of the three-dimensional Nicolai-Samtleben gauged supergravity, in the case of 16 supercharges and local $S O(4) \times$ $S O(4)$ symmetry. This describes the $A d S_{3} \times S^{3} \times S^{3} \times S^{1}$ near-horizon geometry of the double D1-D5 system (references given in $[2,3]$ ). The matter sector of this theory consists of $n$ multiplets each containing 8 scalars and 8 fermions, and the nonpropagating fields are graviton, gravitini, and here also all 12 vector fields. The $8 n$ scalars parametrize the coset manifold $S O(8, n) /(S O(8) \times S O(n))$.

The supergravity Lagrangian is given by

$$
\mathcal{L}=\frac{1}{4} \sqrt{G} R+\mathcal{L}_{\mathrm{CS}}+\mathcal{L}_{\text {kin }}+\sqrt{G} V+\mathcal{L}_{\mathrm{F}},
$$

where $\mathcal{L}_{\mathrm{CS}}$ is the Chern-Simons term for the vector fields, $\mathcal{L}_{\text {kin }}$ is the kinetic term for the scalars, $V$ denotes the scalar potential and finally $\mathcal{L}_{F}$ contains the fermionic terms. In addition to the local $S O(4) \times S O(4)$ gauge symmetry, the theory is invariant under the rigid action of $S O(n)$, rotating the matter multiplets. Specializing to $n=4$ matter multiplets, we consider a global invariance group $S O(4)_{\text {inv }}$ of the potential, embedded as the diagonal of the three $S O(4)$ factors.

Evaluation of the scalar potential $V$ on the two-dimensional space of singlets under $S O(4)_{\text {inv }}$ leads to a potential plotted in fig. 1 . Denote by $\alpha$ the ratio between the couplings of the two factors in $S O(4) \times S O(4)$. The potential depicted in fig. 1 is for $\alpha=1$. The figure exhibits two inequivalent extremal points apart from the local maximum at the origin. The saddle point at $\left(Z_{1}, Z_{2}\right)=(1,0)$ corresponds to a nonsupersymmetric but stable vacuum, as was verified by direct computation of the 


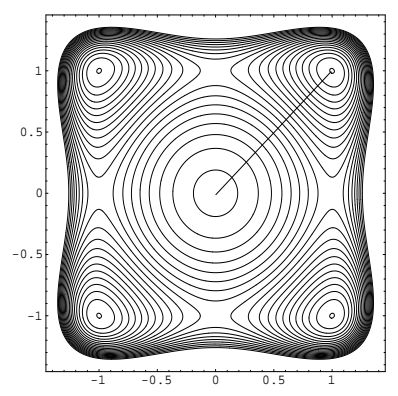

Figure 1.1 Contour plot of the scalar potential $V\left(Z_{1}, Z_{2}\right)$, for $\alpha=1$ [2]. The supersymmetric flow from the central maximum to a local minimum is displayed.

scalar fluctuations around this point [2]. Here I will concentrate on the extremum located at $\left(Z_{1}, Z_{2}\right)=(1,1)$, which preserves $N=(1,1)$ supersymmetry. The flow to this point may be parametrized by a single scalar $Z_{1}=Z_{2}=$ : $(1 / \sqrt{2}) \sinh (Q / \sqrt{2})$.

The ratio of the central charges of the dual conformal field theory at this extremum and that of the CFT at the origin is given through the Brown-Henneaux/Henningson-Skenderis relation between central charge and AdS length, and becomes

$$
c_{\mathrm{IR}} / c_{\mathrm{UV}}=\sqrt{V_{\mathrm{UV}} / V_{\mathrm{IR}}}=1 / 2,
$$

supporting the conjecture that this point corresponds to a mass deformation of the UV conformal field theory, where half the degrees of freedom are integrated out at long distances. The supergravity spectrum around this point can be organized in $N=(1,1)$ supermultiplets, as expected.

With the standard domain wall ansatz $d s^{2}=e^{2 A(r)} \eta_{i j} d x^{i} d x^{j}+d r^{2}$, and changing variables for the domain-wall scalar to the convenient $y=$ $\cosh \sqrt{2} Q$, the first-order equations admit the solution

$$
\begin{gathered}
\frac{(5-y)(y+1)^{2}}{16(y-1)^{3}}=e^{24 g r}, \\
e^{6 A(r)}=\frac{(5-y)^{4}}{128(y+1)(y-1)^{6}} .
\end{gathered}
$$

This solution interpolates between the origin and the supersymmetric extremum, preserving $N=(1,1)$ supersymmetry throughout the flow. By studying linear fluctuations around this analytic solution, one extracts holographic correlation functions along the flow.

Our fluctuations are not hypergeometric, but can all be transformed to an equation known as the biconfluent Heun equation. The two-point 
function of the boundary stress-energy tensor in complex coordinates $z=x^{1}+i x^{2}$ is schematically $\left\langle T_{z \bar{z}} T_{z \bar{z}}\right\rangle=\delta g_{(2)} / \delta g_{(0)}+$ counterterms, and the result is

$$
\left\langle T_{z \bar{z}}(-p) T_{z \bar{z}}(p)\right\rangle=-\frac{k|p|^{4}}{8}\left(\frac{1}{8|p|^{2}}+\frac{1}{2+8|p|^{2}-\Psi_{-2}(p)}\right),
$$

where $\Psi_{-2}(p)$ is the Heun-function analogue of $\psi(p)$ for hypergeometric functions. Unlike $\psi$, however, the coefficient $\Psi$ has no simple closed form, but with some effort the relevant asymptotics can be determined analytically. Taking the infrared $p \rightarrow 0$ limit, the expression (1.5) has the appropriate CFT power-law behavior $p^{2 \Delta_{\mathrm{IR}}-2}$. The correlator is the main result reported in this contribution.

A word about the factor $k$ in the correlator. In the $N=(4,4)$ boundary conformal field theory, $k$ denotes the equal levels of the two $S U(2)^{2}$ current algebras. In terms of the ten-dimensional double D1-D5 supergravity solution with brane charge $N$, the appropriate relation is $k \sim N^{2}$.

Finally, the small distance behavior of the Zamolodchikov $C$ function computed using this and similar correlators is

$$
C_{\text {Zam }}=3 k\left(1-\frac{1}{4 \sqrt{2}}|z|+\mathcal{O}\left(|z|^{3}\right)\right) .
$$

This holographically computed Zamolodchikov $C$-function can then be compared to the "holographic $C$ function", which is simply given in terms of the superpotential $W$ as $C_{\text {hol }}=-3 k / W(Q)$ where $Q$ is the scalar supporting the domain wall, as above. The details of the comparison are left to the references.

\section{Acknowledgments}

It is my pleasure to thank H. Samtleben for collaboration. I would also like to thank the organizers of the Cargèse 2002 ASI. This work was supported by a Marie Curie Fellowship, contract number HPMF-CT-2001-01311, and in part by INFN, by the EC contract HPRN-CT-2000-00122, by the EC contract HPRN-CT-2000-00148, by the INTAS contract 99-0-590 and by the MURST-COFIN contract 2001-025492.

\section{References}

[1] K. Skenderis, Lecture Notes on Holographic Renormalization, Class. Quant. Grav 19 (2002) 5849-5876; hep-th/0209067.

[2] M. Berg and H. Samtleben, An Exact Holographic RG Flow Between 2d Conformal Fixed Points, JHEP 05 (2002) 006; hepth/0112154.

[3] M. Berg and H. Samtleben, Holographic Correlators in a Flow to a Fixed Point, hep-th/0209191. 